\title{
Engineering Geodesy Definition and Core Competencies
}

\section{Journal Article}

\section{Author(s):}

Kuhlmann, Heiner; Schwieger, Volker; Wieser, Andreas; Niemeier, Wolfgang

Publication date:

2014

Permanent link:

https://doi.org/10.3929/ethz-b-000096777

\section{Rights / license:}

In Copyright - Non-Commercial Use Permitted

\section{Originally published in:}

Journal of Applied Geodesy 8(4), https://doi.org/10.1515/jag-2014-0020 


\section{Research Article}

\section{Heiner Kuhlmann*, Volker Schwieger, Andreas Wieser, and Wolfgang Niemeier Engineering Geodesy - Definition and Core Competencies}

\begin{abstract}
This article summarises discussions concerning the definition of "engineering geodesy" within the German Geodetic Commission. It is noted that engineering geodesy by means of its tasks, methods and characteristics is an application-oriented science whose research questions often arise from observed phenomena or from unsolved practical problems. In particular it is characterised by the professional handling of geometry-related problems in a cost-effective manner that includes comprehensive quality assessment at all phases of the problem solution - from planning through measurement to data processing and interpretation. The current methodical developments are primarily characterised by the increasing integration of the measurement and analysis into challenging construction, production and monitoring processes as well as by the transition to spatially continuous methods. A modern definition of engineering geodesy is proposed at the end of this article.
\end{abstract}

Keywords: Engineering Geodesy, Engineering Surveying, self-conception, definition

DOI 10.1515/jag-2014-0020

Received October 14, 2014; accepted October 17, 2014.

\section{Introduction}

The introduction of terms and their common understanding are fundamental to every scientific discipline. Because

\footnotetext{
*Corresponding Author: Heiner Kuhlmann: Rheinische Friedrich-Wilhelms-Universität Bonn Institute of Geodesy and Geoinformation Nussallee 17, 53115 Bonn, Germany, E-mail: heiner.kuhlmann@uni-bonn.de

Volker Schwieger: University of Stuttgart, Institute of Engineering Geodesy, Geschwister-Scholl-Str. 24D, 70174 Stuttgart, Germany Andreas Wieser: ETH Zürich, Institut für Geodäsie und Photogrammetrie, Wolfgang-Pauli-Str. 15, CH-8093 Zürich, Switzerland Wolfgang Niemeier: TU Braunschweig Institut für Geodäsie und Photogrammetrie, Pockelsstraße 3, 38106 Braunschweig, Germany
}

they reflect the area of expertise within the discipline they enable internal communications. However they also provide a platform for communications with neighbouring disciplines. Ever since Helmert (1880) specified geodesy as the "science of measuring and imaging the surface of the earth", many changes of methods, sensors and technology as well as scope of duties and fields of applications of geodesy have occurred. Against this background a discussion of self-conception has taken place within the Section of Engineering Geodesy of the German Geodetic Commission, which covered core competencies and unique features as well as future key research questions and education at university level. This article summarises the discussion and proposes an update to the definition of "engineering geodesy".

We understand engineering geodesy as an application-oriented science, whose research questions often arise from observed phenomena or from unsolved practical problems. Consequently, this contribution covers aspects of both scientifically as well as practically oriented engineering geodesy, in case such a separation is possible or necessary at all.

\section{Historical development of "engineering geodesy"}

Although engineering geodesy is a relatively young geodetic sub-discipline, its definition has been somewhat imprecise, as indicated by Table 1. All definitions are derived from fields of application, with the main focus initially being on civil engineering. Today the spectrum of engineering geodesy applications is far wider. The definition given in [1] is the first one derived not only from measured objects, and in [2] the important bond to other disciplines is first identified explicitly.

The term "engineering geodesy" has started to replace the formerly used term "engineering surveying". The main reason is that the latter often referred only to technical measurements, whereas the former one is broader and is 
understood to cover also the entire set of methodologies for data analysis, modelling of sensors, objects, processes and quality, interpretation and visualisation of results.

\section{Core competencies and unique features}

Methods, processes and characteristic features which distinguish today the activities of engineering geodesy in theory and practice are outlined in the following subsections. The appropriate handling of geometrical questions with end-to-end quality assessment, from planning and measurement to analysis and interpretation, under economic constraints appears to be a primary attribute. The fields of application are almost exclusively located in the interdisciplinary domain, hence the engineering geodesist needs to be competent in high-level processes within his own discipline, but also be knowledgeable of the characteristics and nomenclature of the respective neighbouring disciplines.

\subsection{Reality capture and surveying}

Reality capture, surveying, field measurements [3] or - often used also in a synonymous way - topographic, boundary and as-built surveying refer to geometric and semantic data acquisition and modelling of the current state of an object or area, often also comprising further space-related parameters. The object can be a part of a machinery, a single building, a whole urban district, the slipped mass of a landslide, or an entire mountain range. Such field measurements are usually the basis for creating planning documents and spatial models subsequently required for a construction, fabrication or transformation process.

The measurements may also provide the appropriate information for deciding on the approval of a technical object or deliverable for quality management and accounting. The survey of a current state after completion of the construction or modification process often serves for comparison to the respective target state. This is often described as a check survey.

A recent trend is for survey measurements to be made at all phases of construction, production or transformation operations. This allows documenting and evaluating intermediate steps. The evaluation is often implemented in real-time or with a short time lag. In this context, a thorough understanding of the related processes is needed, in addition to the core engineering geodesy technical compe- tencies. In this respect data acquisition and surveying can be seen as fundamental monitoring activities.

The totality of activities related to surveying measurements, their task-specific and quality-assured planning and analysis as well as the development of the related methods and instruments is an important characteristic of engineering geodesy and clearly represents one of its core competencies.

\subsection{Setting-out}

Setting-out is defined as the transfer of predetermined geometric dimensions from a planning model to the construction site [4]. For example, coordinates or distances are transferred to reality and marked recognisably on site using a feedback-control loop. Setting-out is a core competence and a unique feature of engineering geodesy.

Often high demands are made on relative accuracy of adjacent or nearby elements, e.g. standard deviations of $0.5 \mathrm{~mm}$ have to be achieved for the 3-dimensional settingout of high speed train rails [11]. Today, the measurement processes themselves are highly automated - only the design and installation of the measurement system is generally carried out by experts.

Engineering geodesy is particularly challenged by the setting-out of the alignment of long tunnels where the demands on reliability and accuracy are very high and the working conditions are generally unfavourable. These days the engineering geodesy processes in this context are fully automated and integrated into the building process [12]. Such automation is required because tunnelling is a continuous process that requires real-time geodetic information without interruption, e.g. for controlling the tunnel boring machines or for placing the blast holes. In terms of "engineering navigation" machine control can be seen as the "kinematic generalisation of setting-out" [11].

This form of kinematic setting-out is widely used in road and railway construction, and is increasingly being used for civil engineering. For example, a combination of GNSS and total stations has been developed and successfully used for quasi-kinematic setting-out and for seamless documentation of the building process of very high buildings such as the Burj Chalifa in the United Arab Emirates [16].

Geometric setting-out, the integration of measurement and analysis processes into feedback control loops, and the essential real-time quality management are core elements of engineering geodesy. 
Table 1. Some published definitions of engineering geodesy/surveying.

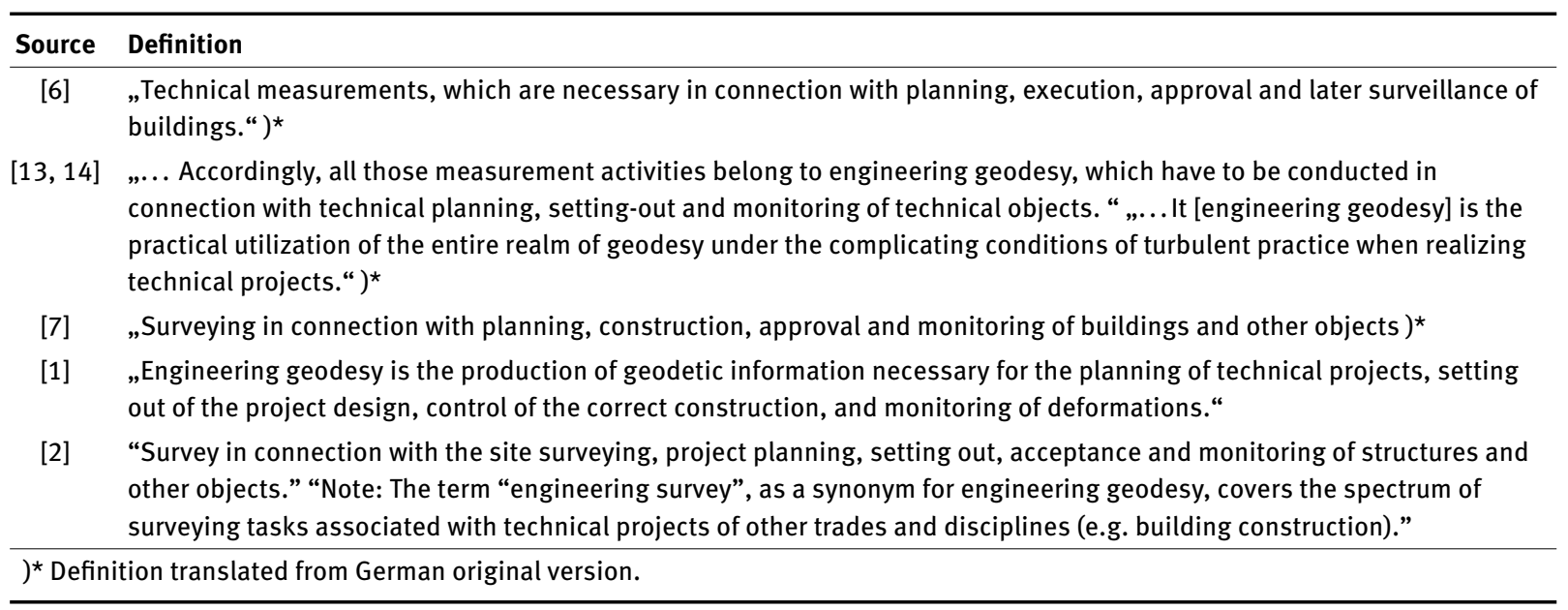

\subsection{Monitoring and its measurements}

In engineering, monitoring generally refers to the data acquisition, observation and supervision [5] of natural and artificial systems. A monitoring system thus allows also intervention, or even control, e.g. if movements do not remain within the defined range of tolerance.

In engineering geodesy, monitoring denotes in particular the metrological registration of the geometric current state of an object, and its comparison to the state in the past. The primary goal is to detect rigid body motion and deformation, and to analyse them in relation to the cause of the changes. For this purpose monitoring networks and/or measurement systems that carefully balance the required information content against various constraints, have to be designed, developed and implemented. They need to be optimised regarding sensitivity with respect to the critical deformations and movements, provide acceptable false alarm rates, be robust, and fulfil all further technical and economic criteria.

For the detection, identification and analysis of changes, engineering geodesy uses a variety of statistical methods, which allow distinguishing point displacements, object movements and deformation while taking into account the uncertainty of all measurements and models.

Current research addresses the transfer of these methods from point- and network-based approaches to areabased ones. Furthermore, dynamic deformation modelling is increasingly being researched, see e.g. [10], which includes both the temporal changes of the observed geometry and the forces causing these changes. This approach builds on the well established characterisation of evaluation models as congruence model, kinematic model, static model and dynamic model. Apart from the treatment of time as a separate dimension, this requires knowledge of the factors generating changes of the monitored objects, as well as at least an approximate knowledge of the transfer behaviour of the object, and thus of dynamic systems. Measurement of the input and output parameters (geometric changes) allows system identification, which in turn permits the drawing of conclusions on the condition of the monitored object. A variety of parametric and nonparametric approaches have been developed to describe the temporal behaviour of monitored objects.

The deformation analysis itself should be carried out in an interdisciplinary manner. Civil engineers, geologists, geotechnical engineers, and members of other allied disciplines, provide information on the dynamic model representation of the object, e.g. the finite element model of a dam. The engineering geodesy core competency is in the integration of such models with actual measurements, e.g. using a Kalman Filter.

Typical applications of engineering geodetic monitoring are the observation and analysis of landslides, ground subsidence, and deformations of structures such as buildings, bridges, dams or tunnels. Besides the determination of the time-evolution of geometric changes, monitoring is also necessary for conservation of evidence. For the geodetic monitoring of natural phenomena, especially changes of the Earth's surface and of the cryosphere, "geomonitoring" is being established as a sub-discipline within engineering geodesy. 


\subsection{Geometry-related phenomena}

In almost every engineering discipline or natural science, measurements are the basis for problem solving and acquisition of knowledge. Often measurements refer to physical dimensions without direct relation to geometry. Engineering geodesy is primarily concerned with geometric parameters such as coordinates, distances, angles and quantities derived from these, e.g. altitude differences, straightness, bending or inclination. However, most engineering geodesy problems also require the determination (and perhaps modelling) of additional spatial parameters, e.g. atmospheric conditions along the signal propagation paths, surface temperature, or material properties. All these parameters and their spatial and temporal variations can be summarised using the term "geometry-related phenomena".

The variations can be associated with the modelled object as well as the measurement system itself, the latter, e.g. with kinematic mapping of objects and whole cities using mobile mapping systems, the former, e.g. with the automatic control of construction machines in tunnelling and both when setting-out from a moving platform.

In the past "spatial discretisation" was an essential method of engineering geodesy, see [1]. Even today there are applications where conclusions referring to a spatial continuum are drawn from measurements of distributed single points [17]. Meanwhile point-wise approaches are often replaced by line-based and areal measurement and analysis methods, which may be referred to as spatially continuous methods. This is realised by sampling with nearly constant, negligibly small discretisation intervals instead of carefully planned single measurements.

Process-oriented approaches to determining and modelling geometry related phenomena play an increasing role. The processes, e.g. related to the construction of a tunnel or a bridge, are observed and the observation results are used partially for the improvement of the process models and partially for process control.

\subsection{Spatial scale: local and regional phenomena}

Engineering geodesy has for the large part been focussed on solving geometry related problems in the field of civil engineering. This is unlikely to change. Nevertheless applications may also be related to machine construction, geotechnics and other allied disciplines. In terms of geodesy, the observed and modelled phenomena therefore often have a local character, but they may also reach re- gional dimensions. Exemplary scale ranges and application fields are:

- $\quad 1-100 \mathrm{~cm}$ : form control for quality management in machine construction; determination of geometry and growth of agricultural crops;

- 10-100 m: setting-out of a family home, structure, surveying of a bridge;

- 1-10 km: installation of a monitoring network; control of a tunnel excavation;

- $\quad 10-1000 \mathrm{~km}$ : mapping of road infrastructure by means of a mobile multi-sensor mapping systems; determination of postglacial and tectonic movements.

Clear demarcation of engineering geodesy with respect to neighbouring disciplines is therefore not possible strictly in terms of spatial scales or geometry-related characteristics only. There is an overlap with state or national geodetic control surveys and physical geodesy at the largest scale, and with geotechnics and mechanical engineering at the lower scales. However, a core competence of engineering geodesy - and a distinction from neighbouring disciplines - is the consistent treatment of geometry-related problems, stretching across several of the above scale ranges, within a single reference frame.

\subsection{Quality assessment and quality management}

Engineering geodesy has always paid particular attention to definition, planning and assurance of the quality of the measurements and analysis results. This is a prerequisite for fulfilling the various requirements of different applications under practical or project-related economic constraints. Simultaneously the risk of consequential errors is limited.

For a long time there has been a focus on accuracy as a measure of quality. The research of modelling, propagation and mitigation of random deviations and their quantification using statistical measures such as standard deviations, confidence ellipses or scalar functions (e.g. the determinant) of covariance matrices in linear or non-linear models, are without doubt core competencies of engineering geodesy. However the evaluation of reliability in the sense of detectability of model errors and of effects of undetected model errors has also been important. Thorough knowledge of measuring instruments and measuring processes, including all relevant technological factors, as well as the redundant acquisition of information using different physical principles, are essential for the evaluation of precision and reliability. 
Calibration and appropriate choice of measurement setups and evaluation processes help to mitigate systematic influences or in some cases even eliminate them. The sensors, measurement processes and analysis procedures have been improved steadily for many fields of application. This applies in particular to the random deviations of the measurements and only to a lesser degree to the systematic ones. A deeper understanding of the measurement procedures as well as the physical sensor models has led to the conclusion that randomising systematic effects to the required degree is not possible or leads to correlations and auto-correlations, which inevitably required the inclusion of stochastic processes into the methodological toolbox of engineering geodesy. The modelling and propagation of accuracy has had to be extended to include systematic contributions to uncertainty. Today greater emphasis is placed on uncertainty modelling, and the "Guide for the Expression of Uncertainty in Measurements (GUM)" [9] is also applied in engineering geodesy for evaluation of the accuracy of measurement systems.

Engineering geodesy is often associated with the capability of - and the presumed disposition to - delivering particularly high measurement accuracy. This does not correspond to the self-conception of the discipline: engineering geodesists measure as accurately as necessary, not as accurately as possible. This integrated consideration of efficiency for the derivation of quality demands from an understanding of the related processes, and the corresponding implementation, are core competencies of engineering geodesy, although not unique features. Nevertheless, it can be argued that engineering geodesy is distinguished within the canon of geodetic disciplines by the fact that it achieves, if necessary, measurements of very high accuracy at local scales. For example, it can define a distance of $1 \mathrm{~km}$ length through special measurement procedures, deterministic and stochastic modelling of different influences with an accuracy of better than $1 \mathrm{~mm}$ [8], or it can adjust components of a particle accelerator with independently developed instruments and procedures relative to each other with an accuracy better than $1 \mu \mathrm{m}$.

Engineering geodesy has early switched to the notion of attribute accuracy, and has developed an extensive set of methods for evaluating, e.g. geodetic networks using a broad quality model which comprises also parameters of sensitivity and separability. Currently, in the context of interdisciplinary collaboration, a comprehensive quality model for application in construction, with appropriate methods for propagation of quality parameters is used [15].

Due to the typical combination of highly technical requirements on the one hand, economic, temporal and local constraints, as well as adverse working and environ- mental conditions, on the other hand, the examination of compliance with quality parameters plays a very important role in engineering geodesy. This control often has to be performed in the field and preferably already while carrying out the measurements. Hence, quality testing can be integrated seamlessly in measurement, evaluation and building processes.

\subsection{Sensor technology and geodetic metrology}

Engineering geodesy is a measurement science. The authors are convinced that engineering geodesy - and also geodesy in general - would lose its relevance for society and science without this metrological component. However, just taking measurements is not at the centre of the metrology competence of engineering geodesy. This competence comprises deterministic and stochastic modelling of the measurement processes, the knowledge of physical sensor models, the acquisition and modelling of the relevant environmental conditions and of all other relevant factors, as well as the indirect determination of required parameters and the quantification of their quality. The data processing with a known system model often leads to a least-squares or maximum-likelihood estimation within a linearised Gauß-Markov or Gauß-Helmert Model. The methodological basis of parameter estimation has been broadened recently to include robust estimators and Bayes estimation, as well as stochastic procedures like Monte-Carlo algorithms or heuristic methods such as genetic algorithms.

Reflecting the variety of application fields and requirements, engineering geodesy relies on a wide range of measurement instruments, sensors and sensor systems. Total stations, GNSS receivers (and antennas), level instruments and terrestrial laserscanners are typically used instruments. Photogrammetic systems, inertial measurement units, optical plummets, hydrostatic levelling systems, gyrotheodolites and lasertrackers are other instruments frequently used in engineering geodesy. Terrestrial microwave interferometers with real or synthetic aperture currently emerge as additions to this instrument toolbox. In addition, engineering geodesists access a pool of sensors, especially in connection with monitoring tasks, such as inclinometers, extensometers, position detectors, fibreoptic strain sensors, or temperature sensors. In connection with calibration, testing and development of sensors, instruments such as laser interferometers, collimators, and others are needed. In particular cases engineering geodesy 
requires the development of new sensors for special applications.

The engineering geodesist needs to design optimal measurement concepts, plan and realise data acquisition, and carry out data analysis with quality control of the results. The necessity for temporal and spatial integration of several sensors and instruments in a multi-sensor configuration can result from these requirements. Multiple multisensor systems can be integrated as redundant or complementary systems, or they can be deployed as spatially distributed sensor networks. Conception, development and calibration of such systems, including their components are key tasks of engineering geodesists. Hence calibration takes a special role. On the one hand it is the requirement for achieving utmost accuracy, as for instance with GNSS measurements with sub-millimetre standard deviation. On the other hand it is increasingly more challenging because measurement systems are becoming more complex and their components may be "black-box" subsystems as far as the operators are concerned. System calibration therefore increasingly replaces component calibration.

\subsection{Reference systems}

Location, orientation and connection of measurements and particularly the subsequent analysis based on coordinates - requires the introduction of appropriate reference systems, where the distinction between observation domain and coordinate domain has to be made [1].

For object volumes of a few cubic metres the reference system can be realised directly and mechanically using a coordinate measuring machine. This is not possible anymore for larger dimensions and the reference frame must be indirectly realised by marked points. The necessity of establishing such reference frames, determining and expressing geometric relations through a link to those frames, and at the same time considering all relevant physical influences - such as deflections of the vertical and geoid heights in the course of staking out a tunnel or particle accelerator - is an important reason why the handling of the geometry-related phenomena mentioned above is not only a core competence, but also largely a unique feature of engineering geodesy.

An additional challenge is often that the points representing the reference frame are not fixed and stable. In fact the frame may change significantly during the project duration through differential movement of marked points or through deformations of the external reference frame. Due to this instability, the acquisition and modelling of moving objects and the increasing combination and integration of several sensors to multi-sensor systems, time as the fourth dimension has become vitally important in engineering geodesy. Well defined and stable reference frames are essential for the modelling of temporal processes.

\section{Summary and concluding remarks}

This article summarises discussions related to the definition of "engineering geodesy" within the section Engineering Geodesy of the German Geodetic Commission. It presents engineering geodesy as an application-oriented science with its own conceptional and methodological approaches that has initially been defined with respect to the measured objects and tasks in connection with civil engineering, but is now increasingly considered as a separate discipline, albeit operating in an interdisciplinary field.

Methods, processes and characteristics, which distinguish the work of an engineering geodesist in practice and science today, have been outlined in Section 3. In summary, the development and optimisation of measurement concepts, setups and data analysis strategies based on a variety of technical and non-technical criteria, and applying theoretical methods as well as numeric simulation and optimisation approaches, are core competencies of an engineering geodesist.

With regard to the actual developments, the following trends can be seen:

- The object to be mapped or monitored is now often not represented by a few carefully chosen individual points but by a point-cloud created using a laserscanner or derived from registered images of digital cameras. The relevant object information is not extracted during the measurement but afterwards during data processing.

- An increasingly close link is realised with photogrammetry, with regards to image processing, object extraction, or orientation and positioning algorithms. In addition, the newest total stations and scanners, or particular add-on systems of laser trackers such as probes and hand scanners, include essentially photogrammetric concepts and solutions.

- The measurement system may no longer be static, but moves along the measured object. This also holds for setting-out, when the planned geometry is transferred to reality directly through a guided or controlled machine without marked waypoints. 
The authors believe that engineering geodesy will further develop and change in future to be continuous in space and time, providing innovative and exciting developments. Based on the above discussion of core competencies and characteristics the authors propose the following new definition of this discipline:

Engineering geodesy is the discipline of reality capture, setting-out and monitoring of local and regional geometryrelated phenomena, paying particular attention to quality assessment, sensor systems and reference frames.

\section{References}

[1] Brunner F. K., On the methodology of Engineering Geodesy, Journal of Applied Geodesy 1 (2007), 57-62.

[2] DIN 18710-1, 2012, Deutsche Norm: Engineering Surveys -Part 1: General Requirements, Beuth Verlag, Berlin.

[3] DIN 18710-2, 2012, Deutsche Norm: Engineering Surveys -Part 2: Site surveying, Beuth Verlag, Berlin.

[4] DIN 18710-3, 2012, Deutsche Norm: Engineering Surveys -Part 3: Setting out, Beuth Verlag, Berlin.

[5] DIN 18710-4, 2012, Deutsche Norm: Engineering Surveys -Part 4: Deformation measurements, Beuth Verlag, Berlin.

[6] FIG, 1971, FIG-Fachwörterbuch, Heft 10 - Ingenieurvermessung. Verlag des Instituts für Angewandte Geodäsie, Frankfurt.

[7] FIG, 1997, FIG-Fachwörterbuch, Band 10: Ingenieurvermessung. Bundesamt für Kartographie und Geodäsie, Frankfurt.
[8] Heunecke O., Auswertung des Ringversuchs auf der neuen Kalibrierbasis der UniBw München zur Bestimmung der Sollstrecken, Allgemeine Vermessungs-Nachrichten 119 (2012), 380-385.

[9] ISO, Guide to the Expression of Uncertainty in Measurement. International Organization for Standardization, Genève, 1995.

[10] Lienhart W., Analysis of Inhomogeneous Structural Monitoring Data, PhD Dissertation, TU Graz, Shaker Verlag, Aachen, 2007.

[11] Möhlenbrink, W., Schwieger, V., 2006, Navigation and quality of construction processes. Proc. XXIII FIG Congress, Munich, Germany, October 8-13.

[12] Niemeier, W., 2006, Geodetic Techniques for the Navigation, Guidance and Control of Construction Processes. In: Proc. 3rd IAG International Symposium on Geotechnical and Structural Engineering and 12th International Symposium on Deformation Measurements, Baden, Österreich, May 22-24.

[13] Rinner K., Über die zunehmende Bedeutung der Ingenieurgeodäsie, Zeitschrift für Vermessungswesen 96 (1971), 209-217.

[14] Rinner, K., Entwicklungstendenzen in der Ingenieurgeodäsie, Allgemeine Vermessungs-Nachrichten (85) (1978), 161-166.

[15] Schweitzer J., Schwieger V., Modeling of Quality for Engineering Geodesy Processes in Civil Engineering, Journal of Applied Geodesy 5 (2011), 13-22.

[16] Van Cranenbroeck J., Advanced Surveying Control Services for Building the Vertical Cities. In: Proc. FIG Working Week 2007, Hong Kong, China, May 13-17, 2007.

[17] Zeimetz Ph., Kuhlmann,H., Use of parametric models for analyzing ground movement measurements in the Rhenish lignite mining area. World of Mining - Surface \& Underground 63 (2011), 256-264. 
